\title{
Linear polarization and composition of VLBI jets
}

\author{
D. Fraix-Burnet* \\ Laboratoire d'Astrophysique de Grenoble, BP 53, 38041 Grenoble Cedex 9, France \\ Received 21 November 2000 / Accepted 10 October 2001

\begin{abstract}
It is shown that linear polarization data can be used to constrain the composition (normal or pair plasma) of pc-scale extragalactic jets. A simple criterion, based on synchrotron and Faraday depolarization properties, is established. It does not depend on the particle density and the length of the emitting region along the line of sight, thus eliminating two physical unknowns.
\end{abstract}

Key words. galaxies: jet - galaxies: active - radiation mechanisms: non-thermal - radio continuum: galaxies polarization - methods: analytical

\section{Introduction}

Due to their synchrotron emission, extragalactic jets are known to contain electrons. But what is the positive charge population: protons or positrons? This makes a huge difference in the kinetic energy to be put in the ejection process and implies different properties for acceleration and propagation mechanisms.

Extracted matter from the accretion disk is comprised of normal (electron-proton) plasma. But accelerating protons to relativistic speeds makes the energetic budget somewhat problematic. The scheme of a two-fluid plasma (Sol et al. 1989; Pelletier \& Marcowith 1998) alleviates this difficulty by reserving the relativistic speed to a pair (electron-positron) plasma, while the bulk of the jet, non relativistic and non radiating, is made of normal plasma.

Observationally, there have been two approaches to determine the nature of the radiating plasma: spectroscopic behavior (Reynolds et al. 1996), and circular polarization (Wardle et al. 1998). The results tend very strongly toward electron-positron pairs for the radiating component.

In this paper, it is shown that linear polarization could also be used to derive the nature of the jet material from very high resolution observations. Based on basic formulae presented in Sect. 2, a very simple analytical criterion is established in Sect. 3. In Sect. 4, some particular observational data are discussed before a brief conclusion is given in Sect. 5 .

\section{Simple properties of synchrotron radiation}

Let us consider an electron population of density $N_{\mathrm{el}}$. The positive charge is assumed to be a mixture of protons

* e-mail: fraix@obs.ujf-grenoble.fr
$\left(N_{\mathrm{pr}}\right)$ and positrons $\left(N_{\mathrm{po}}\right)$. We have $N_{\mathrm{el}}=N_{\mathrm{po}}+N_{\mathrm{pr}}$ and we define:

$\tau=N_{\mathrm{po}} / N_{\mathrm{el}}$.

A simple homogeneous slab with a homogeneous magnetic field is assumed. As explained later on, this assumption is not restrictive, but yields the minimum depolarization that must be observed in a source. We only consider the optically thin part of the synchrotron spectrum.

\subsection{Synchrotron surface brightness and polarization}

The synchrotron surface brightness of a population of electrons (or positrons) with an energy distribution spectral index $p$ is derived from the standard formulae (i.e. (5.43) in Ginzburg 1979):

$I=K_{1}(p)\left(N_{\mathrm{el}}+N_{\mathrm{po}}\right) \gamma_{\min }^{p-1} L B_{\perp}^{(p+1) / 2} \delta^{(p+n) / 2} \nu^{(1-p) / 2}$

with

$$
\begin{aligned}
K_{1}(p)= & 4.48 \times 10^{-11}\left(\frac{p-1}{p+1}\right) \Gamma\left(\frac{3 p-1}{12}\right) \Gamma\left(\frac{3 p+19}{12}\right) \\
& \times\left(8.40 \times 10^{6}\right)^{(p-1) / 2}
\end{aligned}
$$

in Jy $\operatorname{arcsec}^{-2}$, where $\Gamma(x)$ is the Gamma function, $N_{\mathrm{el}}+$ $N_{\text {po }}$ the total radiating particle density, $\gamma_{\min }$ the minimum energy of the electron (positron) population, $L$ the column length along the line of sight in centimeters, $B_{\perp}$ the component of the magnetic field which is perpendicular to the line of sight, $\nu$ the observational frequency, $\delta$ the bulk Doppler factor, $n=3$ for a continuous jet and $n=5$ for a single plasmoid (Lind \& Blandford 1985). 
In the conditions assumed in this paper, the intrinsic polarization of the synchrotron emission is given by (Ginzburg 1979):

$\Pi_{\mathrm{i}}=\frac{p+1}{p+7 / 3}$

which is $\simeq 69 \%$ for $p=2$ and $\simeq 75 \%$ for $p=3$.

\subsection{Faraday depolarization}

The polarized synchrotron radiation emitted by a given set of electrons is affected by Faraday rotation through other sets of electrons along the line of sight. Integration of the light coming from all these sets results in an apparent depolarization called the Faraday depolarization. This is the minimum depolarization that occurs. Any heterogeneity (in density or in magnetic field) within the slab increases the observed depolarization.

For positrons, the Faraday rotation is of opposite sign, so that there is no Faraday depolarization in a pure electron-positron plasma.

For protons, the Faraday rotation is negligible and does not compensate for that of the electrons. Hence, the observed polarization $\Pi_{\mathrm{obs}}$ is constrained by:

$\Pi_{\text {obs }} \leq \Pi_{\mathrm{i}} \frac{\sin (\psi)}{\psi} \leq \Pi_{\mathrm{i}} \frac{1}{\psi}$.

The Faraday rotation angle $\psi$ is given by (Burn 1966):

$\psi=2.35 \times 10^{4}\left(N_{\mathrm{el}}-N_{\mathrm{po}}\right) B_{/ / L \nu^{-2} \delta^{2}}$

where $B_{/ /}$is the component of the magnetic field which is parallel to the line of sight.

\section{Combining surface brightness and polarization informations}

\subsection{Principle}

Both $I$ and $\psi$ are increasing functions of density, $B$ and $L$. The brightest components should then be less polarized in the case of a normal plasma. In principle, this could be an observational test for determining the composition of a jet. However, this is only a statistical argument, based on the improbable assumption that all parameters are equal, particularly the level of homogeneity of the emitting region. Is there another way of translating the incompatibility between high surface brightness and high polarization for a normal plasma?

We propose to use the ratio between the surface brightness $I$ and the Faraday depolarization $\psi$. As calculated in the next section, its notable property is that it does not depend on $N_{\mathrm{el}}$ and $L$ for a normal plasma, eliminating the indetermination regarding these two parameters.

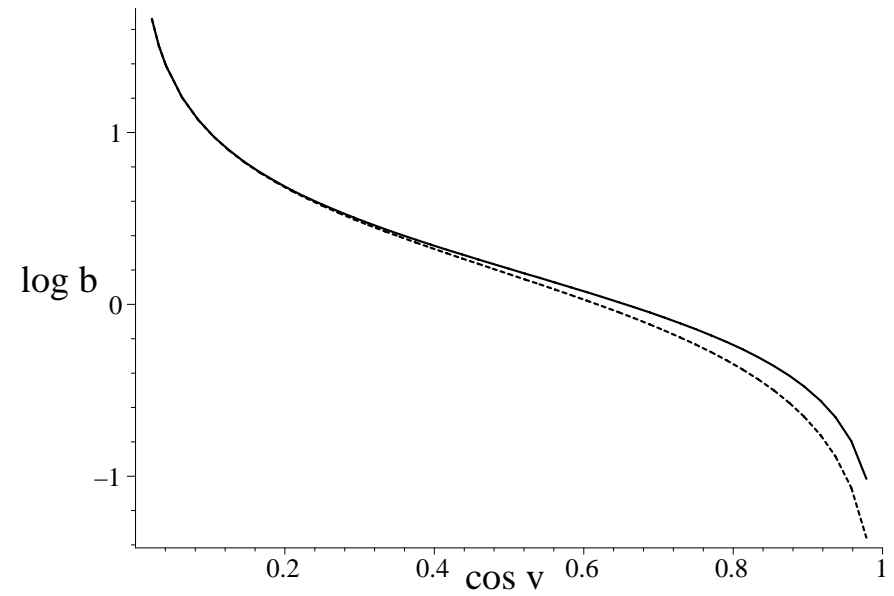

Fig. 1. Function $\log (b(p, v))$ for $p=2$ (solid line) and $p=3$ (dotted line) vs. $\cos v$.

\subsection{Definition of the Brightness Faraday Ratio}

Using Eqs. (2) and (6), we define the "Brightness Faraday Ratio" as:

$B F R=\frac{I}{\psi}=K_{2}(p) \epsilon \nu^{(5-p) / 2} \gamma_{\min }^{p-1} \frac{B_{\perp}^{(p+1) / 2}}{B_{/ /}} \delta^{(p+n-4) / 2}$

where

$K_{2}(p)=K_{1}(p) / 2.35 \times 10^{4}$,

in $\mathrm{Jy} \operatorname{arcsec}^{-2} \mathrm{rad}^{-1}$, and

$\epsilon=\frac{\left(N_{\mathrm{el}}+N_{\mathrm{po}}\right)}{\left(N_{\mathrm{el}}-N_{\mathrm{po}}\right)}=\frac{1+\tau}{1-\tau}$.

The function $\epsilon$ essentially $\approx 1$, except for a nearly pure pair plasma in which case it can be much higher. Let $v$ be the angle between the magnetic field and the line of sight. It becomes:

$\frac{B_{\perp}^{(p+1) / 2}}{B_{/ /}}=B^{(p-1) / 2} b(p, v)$

with

$b(p, v)=\frac{(\sin v)^{(p+1) / 2}}{\cos v}$.

The function $b(p, v)$ is plotted in Fig. 1. It is smaller than 0.1 for $v \leq 15^{\circ}$ and is higher than 10 for $v \geq 85^{\circ}$.

Finally, $B F R$ can be written in the following way:

$B F R=K_{2}(p) \epsilon b(p, v) \nu^{(5-p) / 2} \gamma_{\min }^{p-1} B^{(p-1) / 2} \delta^{(p+n-4) / 2}$.

\subsection{Numerical value for pc-scale jets}

The determination of the physical parameters in extragalactic jets is a major problem. We now re-write Eq. (12) in a convenient form to numerically apply it to jets.

As suggested by the observations, $p$ is somewhat between 2 and 3: $K_{2}(2)=0.103$ and $K_{2}(3)=0.074$. Even if it is a somewhat uncertain guess, a typical value for 
the magnetic field appears to be $B \approx 10^{-2}$ G. $\gamma_{\min }$ seems to be more constrained at about $10^{2}$ (see discussion in Reynolds et al. 1996). The allowed variations of these two parameters are probably not much higher than a factor of 10 in most cases.

Defining:

$\nu_{10}=\left(\frac{\nu}{10^{10} \mathrm{~Hz}}\right), \quad \gamma_{100}=\left(\frac{\gamma_{\min }}{10^{2}}\right), \quad B_{-2}=\left(\frac{B}{10^{-2} \mathrm{G}}\right)$,

we obtain:

$$
\begin{aligned}
& B F R_{p=2}=4.06 \times 10^{4} \epsilon b(2, v) \delta^{(n-2) / 2} \nu_{10}^{3 / 2} \gamma_{100} B_{-2}^{1 / 2}, \\
& B F R_{p=3}=1.29 \times 10^{4} \epsilon b(3, v) \delta^{(n-1) / 2} \nu_{10} \gamma_{100}^{2} B_{-2}
\end{aligned}
$$

Since $B F R$ is intended to test the normal plasma hypothesis, one can assume $\epsilon \approx 1$ (see Sect. 3.2).

The value for $n$ depends on the geometry of the source (see Sect. 2.1). However, jets are characterized by a complex continuous structure so that most probably: $n=3$. This makes a relatively softer dependence on the Doppler factor than for $n=5$.

This Doppler factor is known from apparent motions in quite a lot of jets. It seems to be rarely higher than about 10 (i.e. Zensus 1997).

The most uncertain parameter in the expression of $B F R$ is undoubtedly the angle $v$ which cannot be easily determined from the observations. However, as said previously (see Fig. 1$), b(p, v)$ is higher than 10 only when $v$ is larger than $85^{\circ}$ (and $b(p, v)>5$ when $v>80^{\circ}$ ), that is when the magnetic field is nearly exactly in the plane of the sky. In practice, by comparing a given set of several structures within a jet and/or in different sources, it would certainly be possible to restrain $v$ to a reasonable range where the function $b(p, v)$ can be given a representative upper limit.

Finally, the choice of $p$ between 2 or 3 might not be so critical.

\subsection{A criterion for the composition of jets}

For a given set of physical parameters, $B F R$ is the maximum value allowed for $I / \psi$ in the case of an electronproton plasma. Combining Eqs. (5) and (7) the scheme is therefore very simple:

$\frac{I_{\mathrm{obs}} \Pi_{\mathrm{obs}}}{\Pi_{\mathrm{i}}}>B F R \Rightarrow$ normal plasma excluded

where $I_{\mathrm{obs}}$ is the observed surface brightness.

To avoid the contribution of smearing in the observing beam that can particularly affect the observed polarization and also the intensity, the highest spatial resolution is recommended. As a consequence, the VLBI observations are expected to provide the most interesting data.

Note that criterion (14) can be used with other criteria like those in Reynolds et al. (1996) to reduce uncertainties on parameters.

\section{Comparison with observations}

In this section, we illustrate the use of the $B F R$ criterion with observations. A concrete application of the proposed criterion requires a thorough study of individual cases or of a statistically significant sample, in order to determine the most appropriate values for the parameters in $B F R$.

\section{1. $M 87$}

We start with the example of knot $\mathrm{A}$ in the jet of $\mathrm{M} 87$ at 0.14 arcsec resolution and $15 \mathrm{GHz}$ as observed by Owen et al. (1989). The surface brightness from their map is found to be about $15 \mathrm{Jy} \operatorname{arcsec}^{-2}$ with a $\approx 30 \%$ polarization. This yields:

$\frac{I_{\mathrm{obs}} \Pi_{\mathrm{obs}}}{\Pi_{\mathrm{i}}} \approx 6.5$.

In this case, it is not possible to draw conclusions about the composition of the jet, but at this resolution, beam smearing is quite certain and the use of the criterion is somewhat hopeless. Taking the observations by Junor et al. (1999) at $43 \mathrm{GHz}$ and 0.33 mas $\times 0.12$ mas resolution, we obtain a surface brightness of about $10^{6} \mathrm{Jy} \operatorname{arcsec}^{-2}$. Assuming a polarization of $10 \%$, this would give $I_{\mathrm{obs}} \Pi_{\mathrm{obs}} / \Pi_{\mathrm{i}} \approx 1.4 \times 10^{5}$. Preserving a normal plasma hypothesis would put some interesting contraints on the parameters in Eq. (13). This illustrates the usefulness of the criterion presented in this paper when applied to very high resolution data.

\section{2. $3 C 273$}

In Lister \& Smith (2000), both surface brightness and polarization of several components in different sources are compiled. For component $\mathrm{G}$ of the jet in $3 \mathrm{C} 273$ we derive a surface brightness of about $5 \times 10^{5} \mathrm{Jy}_{\operatorname{arcsec}}-2$ and a rather low polarization of $4 \%$. However, this still yields at $22 \mathrm{GHz}$ (resolution of 0.97 mas $\times 0.38$ mas):

$\frac{I_{\mathrm{obs}} \Pi_{\mathrm{obs}}}{\Pi_{\mathrm{i}}} \approx 3 \times 10^{6}$.

In components $\mathrm{C}$ and $\mathrm{D}$, we have a surface brightness of about $4 \times 10^{5} \mathrm{Jy} \operatorname{arcsec}^{-2}$ with about $21 \%$ polarization. This yields:

$\frac{I_{\mathrm{obs}} \Pi_{\mathrm{obs}}}{\Pi_{\mathrm{i}}} \approx 10^{5}$.

These high observed ratios require, for a normal plasma, high values of the parameters (i.e. $n=5$, large Doppler factor, high value for $b(p, v) \ldots)$.

One way to reduce some uncertainties on the parameters would be to compute the above ratio in every structure of the jet. For instance, it would be hard to believe that $v$ is the same everywhere in the jet, hence it would be possible to put a global upper limit on $b(p, v)$. Note also that the composition of the radiating material is certainly the same everywhere in a given jet. 


\section{3. $3 C 279$}

From the same paper (Lister \& Smith 2000), we find that component $\mathrm{F}$ in the jet of $3 \mathrm{C} 279$, at about the same resolution, has a surface brightness of about $1.4 \times 10^{7} \mathrm{Jy} \mathrm{arcsec}^{-2}$ and $18 \%$ polarization. This yields:

$\frac{I_{\mathrm{obs}} \Pi_{\mathrm{obs}}}{\Pi_{\mathrm{i}}} \approx 3.6 \times 10^{6}$.

This again shows that our criteria provide interesting constraints on the parameters in Eq. (13) if one wishes to assume a normal plasma. The fact that the ratio found in knot $\mathrm{F}$ of $3 \mathrm{C} 279$ is close to that for knot $\mathrm{G}$ in $3 \mathrm{C} 273$ is undoubtly pure chance, but comparing values found in several jets is another way to use the $B F R$ criterion and find hints for the composition of jets in general.

\section{Conclusion}

The present study has been initiated from the synchrotron emission simulations (Despringre \& Fraix-Burnet 1997; Fraix-Burnet in prep.) in which it appears nearly impossible to reproduce both surface brightness and polarization of typical VLBI jets. We have then devised a simple tool, presented in this paper, to constrain the composition of a jet with linear polarization data.

It is based on the Faraday depolarization which is the minimum depolarization that occurs from a normal plasma. This yields a criterion, easily computed from the observations, that does not depend on the length of the emitting plasma along the line of sight nor on density of the emitting particles. These two parameters are always very difficult to estimate.

Still, there are a few uncertain parameters in the criterion, but from a few illustrative examples presented in this paper, it already appears that they should be pushed to high values if one assumes a normal plasma. A complete statistical study of several jets or a very detailed study of a well-observed jet is necessary to reach more significant conclusions. This is beyond the scope of the present paper.

Our criterion is not valid in the self-absorbed part of the spectrum. It is certainly not a problem for frequencies above $10 \mathrm{GHz}$ in the jets, but it should be applied to cores only with caution.

\section{References}

Burn, B. J. 1966, MNRAS, 133, 67

Despringre, V., \& Fraix-Burnet, D. 1997, A\&A, 320, 26

Junor, W., Biretta, J. A., \& Livio, M. 1999, Nature, 401, 891

Ginzburg, V. 1979, Theoretical Physics and Astrophysics (Pergamon Press)

Lind, K. R., \& Blandford, R. D. 1985, ApJ, 295, 358

Lister, M. L., \& Smith, P. S. 2000, ApJ, 541, 66

Owen, F. N., Hardee, P. E., \& Cornwell, T. J. 1989, ApJ, 340, 698

Pelletier, G., \& Marcowith, 1. 1998, ApJ, 502, 598

Reynolds, C. S., Fabian, A. C., Celotti, A., \& Rees, M. J. 1996, MNRAS, 283, 873

Sol, H., Pelletier, G., \& Asseo, E. 1989, MNRAS, 237, 411

Wardle, J. F. C., Homan, D. C., Ojha, R., \& Roberts, D. H. 1998, Nature, 395, 457

Zensus, J. A. 1997, ARA\&A, 35, 607 